du 10 juin 1936 (Compt. Rend. Acad. Agriculture France, 22, page 673, 1936).

En matière d'ensilage, l'absolutisme n'est point conseillable. Il faut avoir liberté de choisir le système d'ensilage qui convient le mieux suivant les localités, les conditions climatiques, la qualité du fourrage, la destination du fourrage, la potentialité économique, la main-d'œuvre et plusieurs autres circonstances. Un seul absolutisme s'impose : Il doit s'agir d'un ensilage lactique, tel qu'il ressort de nos recherches bactériologiques (1907) qui ont reçu dernièrement une confirmation par la "J. Jealott's Hill Research", Station de Bracknell (Berks), et le Laboratoire de Bactériologie agricole de l'Université de Reading (Journ. Agricult. Science, 27, avril 1937, 271-et 294).

\title{
UNE THÉORIE PHYSICO-CHIMIQUE DE L'ALLAITEMENT ARTIFICIEL
}

\author{
par
}

\section{Dr L. PIKLER}

Ancien assistant à la "Comte Apponyi " Policlinique, Budapest (Hongrie).

Pour expliquer les différences existant entre la mortalité et la morbidité des nourrissons allaités au sein et alimentés au biberon, on faisait, au cours des temps, intervenir des causes diverses.

Une des plus anciennes interprétations à ce sujet est celle de J.-J. Rousseau, qui, dans son "Émile", chef-d'œuvre de propagande pour répandre de plus en plus la pratique de l'allaitement naturel, confronta le "naturel " et l'" artificiel " en décidant en faveur du premier, méthode naturelle, en cherchant dans les déviations de la Nature les causes responsables de l'incompatibilité du second.

La validité de sa thèse concernant la supériorité de l'allaitement au sein se confirmait au cours des temps d'une facon incontestable [33]. Toutefois, en ce qui concerne les arguments de ce philosophe, énoncés sous la forme la plus emphatique, les dernières conclusions relatives à la priorité prétendue du naturel nous semblent à l'heure actuelle - du moins en ce qui concerne l'allaitement infantile avoir beaucoup perdu de leur persuasivité.

Sans doute, en abordant la question par les méthodes purement médicales, par les moyens impartiaux de la chimie, de la physique, de la microbiologie, etc., on ne tarde jamais à admettre la supériorité évidente du lait maternel. Néanmoins, il faut également reconnaître que l'administration du lait de vache, dans son état naturel, s'accompagne d'inconvénients chez les nourrissons soumis à ce régime et que ce sont inversement les diverses modifications apportées au lait 
de vaches qui l'approprient - en le "dénaturant" - aux besoins infantiles, à l'encontre de toutes spéculations philosophiques.

Est-ce la " déviation de la nature " qui doit y être accusée ? Or, un examen détaillé de ce problème nous autorisera à contester la responsabilité prétendue du caractère "dénaturatif " des modifications apportées au lait de vache, et - pour expliquer l'incompatibilité d'un régime lacté quelconque -, il conviendra d'envisager les méthodes opératoires pratiquées dans le cas en question.

Une appréciation impartiale des diverses méthodes de l'alimentation nous conduira en effet à la conclusion, que l'allaitement artificiel est en train de perdre son caractère autrefois fâcheux et - pourvu que l'alimentation soit bien eonduite -, la possibilité d'atteindre les bons résultats réservés autrefois aux nourrissons au sein, n'est plus une chimère. Au surplus, l'amélioration notable, qui se présente dans les pays urbanisés et industrialisés va persister dans son intégrité, malgré que l'allaitement maternel y soit de plus en plus remplacé par l'allaitement artificiel.

Ces conquêtes de la diététique infantile tiennent d'une part à la propagation de l'hygiène générale. D'autre part cependant, ce sont diverses disciplines spéciales qui ont le mérite d'avoir retrouvé la technique convenable des corrections nécessaires dans la composition du lait de vache, soit à l'aide des interventions chimiques, soit en appliquant les nouvelles découvertes de la physique, de la microbiologie, etc.

Les tendances récentes de la diététique infantile font appel aux méthodes de la chimie physique. Les recherches en cette direction caractérisent les changements d'état colloïdal des protéines comme fonction de leur milieu ionique [74]. Ce sont particulièrement les études approfondies de Jacques LoEB [52], de ses collaborateurs et successeurs qui ont le mérite de nous avoir mis en lumière les circonstances de ces altérations.

La vérification expérimentale de la théorie de DoNnAN, ainsi que les applications diverses de celle-ci, réussirent à féconder non seulement les domaines de la biochimie purement scientifique, mais aussi la science laitière appliquée [74], notamment la technologie du lait, du beurre, du fromage, de la caséine, etc. En dehors de son importance pour l'industrie laitière, cette nouvelle conception des protéines, - qui semble jusqu'ici loin d'être définitivement exploitée-, est d'un intérêt capital pour l'alimentation des nourrissons.

Au point de vue de la chimie physique le lait a les caractères d'une suspension colloïdale, d'une solution vraie, enfin, d'une émulsion. Les protéines s'y trouvent dispersées dans une solution qui 
renferme des molécules et des ions, le tout constituant un système "tampon».

Le composant protéique essentiel du lait de vache est la caséine, laquelle est accompagnée par des quantités faibles d'albumine et de globuline. Dans le lait de femme, par contre, ce sont les dernières. qui prédominent.

La caséine et les autres substances protéiques forment selon leur quantité, leur qualité et selon leur état actuel, un système de protection. L'étude de cet édifice suivant Ch. Porcher [74] nous fait eomprendre la tenue des protéines du lait vis-à-vis du chauffage, de l'emprésurage, de l'acidification spontanée et provoquée, etc. Nos connaissances relatives à la structure colloïdale du lait sont d'une grande importance pour l'industrie, pour le contrôle du lait et pour les problèmes de l'allaitement.

En ce qui eoncerne les connaissances sur le lait à l'origine, nous ne les prendrons que comme points de départ pour notre discussion. Plus importante est l'étude des altérations et des décompositions d'ordre physico-chimique effectuées par les manipulations usuelles pratiquées à la cuisine et dans la technique.

Les agents essentiels à citer sont en premier lieu les opérations physiques.

Déjà l'a bandon du lait au repos pendant quelques heures engendre quelques modifications colloïdales, en dehors de l'acidification spontanée. Les agents physiques les plus usités, à noter, sont : l'agitation [4], les moyens divers de l'écrémage, puis la chaleur $[3,62,74,104]$ et le froid, les irradiations [21], le courant électrique [72] - etc.

Les agents chimiques à noter sont : l'addition d'eau au lait [104], l'addition d'acides [104], celle d'alcalins (rattrapage), l'addition de sels, - particulièrement de ceux de Ca, auparavant parfois de citrates - , des cristalloïdes (sucrage), des colloïdes (tels la saccharose, les farines dextrinisées, maltées [54], bouillons de gruau, d'orge, de riz, d'avoinê), l'addition de protéines desséchées, de gélatine [98], de gomme, etc.

A la série des agents proprement physiques et chimiques s'ajoutent les facteurs biologiques, en premier ordre les actions fermentatives : celles de la présure [74], de la lactase, de la pepsine [25] et d'autres protéases, enfin de la lipase propre du lait et de la lipase $[20,4]$ de l'estomac $[25]$.

Tous ces agents physiques, chimiques et microbiens altèrent le lait de différentes façons. Ce sont particulièrement les altérations du milieu ionique, - particulièrement celles de la réaction -, puis celles de l'état colloïdal des protéines, qui vont nous occuper dans notre présent exposé. 
L'étude du système des électrolytes comprend les questions de l'abaissement cryoseopique, de la réfraction, de la conductibilité, du transport électrique, etc. Les questions qui sont particulièrement intéressantes, sont celles des changements de l'acidité actuelle et potentielle (titrable), puis les questions concernant le tamponnement. L'étude de ces questions ne se borne pas au lait simple mais vient d'être complétée par des recherches portées sur le tamponnement des nourritures infantiles préparées, des mélanges divers, des coupages avec des décoctions différentes, des babeurres, des laits albumineux, etc. $[64,16,38]$.

Les recherches concernant les changements de l'état colloïdal des protéines comprennent également une multitude de questions : celles de la dispersion, de l'hydratation, des charges électriques, de la tension superficielle [4,5], de la viscosité [103], du gonflement, de la pression osmotique, de la dialyse, des potentiels de membrane, des équilibres de Donnan, etc. [52].

Cette énumération détaillée suffit à démontrer la grande diversité des questions d'ordre purement physico-chimique.

Or, on peut bien supposer que dans la série de ces altérations citées on en trouvera certaines qui sont désirables pour la diététique infantile et d'autres qui ne le sont pas. La question se pose alors : quelles altérations sont à réaliser et quelles sont celles à éviter?

Plusieurs auteurs attribuent une importance spéciale aux changements du milieu ionique. Il y a des ehercheurs, comme FriedenTHAL [24], qui veulent équilibrer le rapport des ions mono- et bivalents. Quelques auteurs modernes, - comme Mo. KIM, MARRIotT [56], etc. - accordent une importance particulière à l'abaissement relatif du tamponnement. Or, ce seraient les babeurres et ses eompléments, les laits acidifiés divers, très répandus dans la diététique infantile, qui atteindraient ce but, en assurant un milieu, un $p \mathrm{H}$ plus convenable aux fonctions peptiques, lipolytiques, bactériologiques, aux fonctions motrices, etc. [27, 109].

Certains autres auteurs soutiennent - également en relation avec les laits acidifiés, mais à l'inverse de cette opinion - l'importance spéciale des modifications subies par les protéines (MARFAN [55], Moll [61], Brennemann [7]) sans en préciser toutefois le caractère. On peut soupçonner que les changements susdits du milieu ionique sont alors à considérer comme conditions déterminantes de l'état colloïdal des protéines. Ces changements, - bien connus d'après les recherches de L. Loeb $[52,41]$, Donnan, etc. varient surtout avec le $p \mathbf{H}$, ainsi qu'avec la valence et la qualité des ions en question, lesquels facteurs jouent aussi un rôle.

L'application de quelques procédés physiquès nous donne des renseignements précieux sur l'état colloïdal des protéines en ques- 
tion. Les modifications colloïdales susdites sont à étudier par l'examen du degré de la dispersion, de l'hydratation, des charges électriques, de l'adsorption, de la viscosité [103], de la pression osmotique des protéines $[46,51,52]$, des potentiels de membrane, ete. Ces facteurs varient dans un sens concordant. Au cours de ces variations, on trouve toujours une certaine acidité, où les charges électropositives et électrónégatives se compensent. Au point précis, que l'on a désigné point isoélectrique $[41,51,52]$, non seulement le passage des particules et leur transport électrique est supprimé entre des électrodes dans un bac à électrophorèse, mais aussi les autres indices caractéristiques revêtent des valeurs extrêmes. Citons l'hydratation, l'affinité chimique, la viscosité, la pression colloïdo-osmotique, lesquelles s'abaissent aux valeurs minima, tandis que la tension superficielle et la labilité colloïdale (e'est-à-dire la floculabilité) atteignent des valeurs maxima. Les protéines étant dépourvues à ce point de leur pouvoir absorptif et faute de l'affinité chimique, la minéralisation va s'abaisser au minimum [74].

C'est au point isoélectrique que les colloïdes perdent une partie considérable de leur eau fixée [9], et que la caséine-sol se convertit en caséine-gel en perdant son aspect hydrophile en émulsion et prenant le caractère hydrophobe en suspension [102]. A ce point remarquable, le gonflement devient minimum et - en conséquence le rapport de l'eau libre à l'eau fixée atteint des valeurs maxima.

Habituellement; ce sont les substances dissoutes qui présentent de l'intérêt dans l'alimentation infantile et non pas le dissolvant lui-même. En considérant cependant la portée extraordinaire de l'eau en diététique infantile, on peut bien se demander quelle importance on doit attacher, dans les laits préparés, aux changements manifestés par la dernière, en sus de la signification des électrolytes soulignées déjà par quelques auteurs et en sus de l'im. portance des modifications colloïdales mises en évidence par les autres.

Il est singulier de noter le silence de la littérature pédiatrique pour préciser l'allure des changements des protéines du lait au point de vue colloïdal ; elle se borne à l'emploi commode des expressions devenues en effet courantes et elle se sert de généralités, comme " la finesse de la dispersion, la voluminosité des grumeaux, des caillots, des flocons, etc, ”, en même temps que la théorie récente des colloïdes ne tarde plus à déterminer d'une façon exacte les altérations survenues sous l'action d'un apport acide quelconque.

En effet, on sait bien que les altérations subies par les matières colloïdes sont toujours liées aux changements relatifs à la fixation de l'eau. Les transformations des substances dissoutes comprennent et expriment alors - dans un sens réciproque - toujours celles 
du dissolvant. On peut donner, en quelque sorte, un rôle immédiat et autonome aux changements d'état de l'eau, qui suivent les altérations subies par les substances dissoutes.

Les altérations effectuées dans le milieu ionique par l'acidification sont responsables des altérations protéiques, lesquelles conditionnent - de leur part - une altération dans le rapport $\frac{\text { eau libre. }}{\text { eau fixée. }}$

De cette manière, la répartition de l'eau libre et de l'eau fixée, va être conditionnée, dans les solutions collö̈dales, indirectement, par les variations primaires du milieu ionique.

Or, on reconnaît depuis longtemps l'importance primordiale de l'eau pour les nourrissons [55, 77], même en comparaison des matières calorigènes dissoutes, puisque l'on sait fort bien que les nourrissons supportent le jeûne toujours incomparablement mieux que la privation d'eau. Les troubles alimentaires aigus et chroniques se traduisent - par suite de l'imperfection du mécanisme osmorégulateur - toujours par des désordres de l'échange hydrique, soit par des symptômes adéquats relatifs à la fixation de l'eau (effets " hydropigènes ", "exsiccogènes "), soit par des troubles de l'élimination de cette eau [43], (urines, selles, perspiration insensible). La physiologie de l'alimentation est en train d'explorer les détails de ce mécanisme compliqué. Ce que l'on peut dire aujourd'hui de certain c'est que l'essentiel de ces troubles alimentaires est toujours un relâchement dẹ la conjonction intime de l'eau initialement fixée aux colloïdes des humeurs, des cellules, des tissus, et que ces désordres se manifestent toujours par un certain ébranlement du bilan hydrique.

Il va de soi que l’organisme - étant doué de régulations compensatrices - va fréquemment récupérer les grandes pertes d'eau et, grâce à cette défense souvant suffisante, on omettra les signes manifestes d'une déshydratation quelconque dans le cas des diarrhées légères, Les régulations vicariantes entrées en jeu, lesquelles présentent quelque souplesse dans leur fonctionnement, montrent, dans l'élimination de l'eau, une économie marquée dans les cas de déperditions aqueuses par les selles diluées, soit dans la diminution de l'émonction urinaire, soit par la diminution de la perspiration invisible : Cfr. le schéma de J AHR [42].

Le travail suggestif de Rockemann [84] - conforme d'ailleurs à quelques travaux antérieurs [89], à ce sujet, - nous fournit une démonstration expérimentale de la possibilité de l'augmentation des éliminations urinaires, sous l'influence d'un régime de babeurre qui se manifeste simultanément par un accroissement parallèle du nourrisson en poids. Il ressort, de ces observations, que cet excédent d'urine - en présence d'une fixation croissante de l'eau, 
laquelle entraîne l'augmentation en poids - n'est pas à expliquer autrement que comme un simple signe de la surabondance de l'eau disponible pour l'organisme.

Si l'on regarde de près la nature intime de l'état colloïdal, on ne tarde pas à apercevoir que celui-ci n'est qu'un état d'équilibre intermédiaire, instable, même "improbable", dans le sens de la thermodynamie, entre les solutions vraies, stables, et l'état de dispersion grossière. Il manque peu de chose pour que les énergies superficielles, liées aux charges électrostatiques [65], se déchargent à la manière des condensateurs électriques, - lequel processus va s'accompagner de la déshydratation des colloïdes lyophiles en dispersion.

Cette compensation des charges électriques se produit déjà dans le lait au repos et presque chaque intervention appliquée mènera à une diminution de l'eau fixée aux colloïdes. Cette déshydratation des colloïdes correspond toujours à une diminution de la finesse de la dispersion. La dispersion, ce qui correspond à l'hydratation des micelles de la caséine, est d'ailleurs déjà initialement différente (The Svedberg) et varie d'après l'espèce bovine. (KostukR, cfr. Samuelsson [87].) Chaque diminution dans la finesse de la dispersion se manifeste par une diminution de la quantité de l'eau "solvante" et dans une augmentation consécutive de "l'espace solvant réel » (POLANYI [32]), ce qui fait comprendre que la pression osmotique des colloïdes va s'abaisser et s'annihiler selon les variations déshydratantes corrélatives.

Le degré de la dispersion el celui de la pression osmotique des protéines sont, en rapport inverse, étroitement liés l'un à l'autre. En dehors de cela, l'allure de la tension superficielle des protéines, de la viscosité et, par excellence, celle du potentiel de membranes varient dans le même sens que les deux degrés en question. (Déshydratation) [40.52]. Nous disposons done d'une multiplicité de moyens indirects convenables pour une appréciation de la pression osmotique des protéines.

La question de l'allure de l'hydratation et du degré de la dispersion a fait d'ailleurs l'objet d'examens directs tyndallométriques et de quelques autres recherches analogues, par rapport à la caséine (Holw ERDA, SoHneck [92]). En faisant appel aux diverses méthodes directes et indirectes dont nous venons de citer quelques-unes, ces altérations de l'hydratation et la dispersion sont parfois aisées à suivre (par exemple, par le nombre d'or, le nombre d'alcool) [59].

Toutes ces méthodes illustrent le fait qu'au point isoélectrique de la protéine, la valeur de l'eau libre, c'est-à-dire de l'eau disponible pour les besoins osmotiques des sels, atteint une valeur maximum. 
A ce point là, les " anomalies » dues au contenu protéinique de la solution vont disparaître. A ce point "isoélectrique », la pression colloïdo-osmotique (oncotique) s'abolit en s'abaissant à sa valeur minimum.

On sait que la pression osmotique des protéines est déjà primitivement inférieure à celle des cristalloïdes. Au surplus, - conformément à la théorie de Donnan-Loeb - leur propre pression, presque nulle, est négligeable. Et c'est plutôt la distribution spéciale des sels ionisés qui rend possible la pression osmotique réelle.

Mais, en ce qui concerne la propriété la plus essentielle de la pression osmotique, c'est-à-dire la capacité à attirer l'eau [1], celleci subsiste toujours. Cette tendance est toujours incomparablement plus grande que l'on ne persait à la base de l'abaissement cryoscopique et dépasse largement cette valeur. Cet afflux aqueux [41] est bien connu depuis les recherches mémorables de Starling. Les exemples simples d'Isvesco [41] illustrent en effet d'une façon suggestive la déshydratation d'une solution saline, effectuée par une solution colloïdale mise en contact avec le premier au moyen. d'un sac dialyseur.

C'est cette pression osmotique des colloïdes que l'on peut faire intervenir dans la théorie du développement et de la disparition des exsudats, transsudats et oedèmes (FARKas, Rusznyak, Govaerts, ACHARD [9]).

De même façon, les colloïdes - comme substances mal diffusibles - jouent un rôle capital dans l'action des purgatifs. On sait déjà depuis longtemps que les substances mal diffusibles (y compris les colloïdes) empêchent la résorption de l'eau au niveau des intestins. en entretenant une succulence permanente des contenus intestinaux. Ce ralentissement de la résorption de l'eau dont nous avons précédemment parlé est d'abord à noter à la suite de l'ingestion de solutions concentrées salines mal diffusibles (par exemple le sulfate de magnésium), puis après l'administration de quelques autres subsstances mal diffusibles, même en faibles doses (par exemple la phénolphtaléine de VAMOSSY) pourvu que l'on surpasse la tension partielle des protéines du sang (RHorer). Si ce phénomène est conditionné par la "lyophilie " d'un colloïde, le ralentissement de la résorption des sucs va varier avec l'état actuel de la protéine. Le pouvoir osmo-laxatif étant lié à l'état osmo-actif de la protéine, il sera utile d'envisager les facteurs qui l'influencent.

La pression osmotique des solutions ioniques varie avec la concentration des matières dissoutes, laquelle concentration définit le nombre des particules osmo-actives entrant en jeu. Toutefois, ce rapport ne vaut que pour les solutions étendues puisque les solutions salines concentrées ainsi que les solutions colloïdales n'obéissent. 
plus en général aux lois de $V_{A N}$ ' $t$ Hofr. C'est pourquoi la concentration des protéines et leur poids "moléculaire», "micellaire", ne peuvent être considérés que comme points de repère. Néanmoins, - même si la pression augmente plus fortement que la concentration [I] [46], on peut, du moins, apprécier l'allure des changements provoqués. Or, en additionnant de l'eau à une solution colloïdale quelconque, il nous sera peut-être loisible de compter, en pareilles conditions, sur un abaissement de la pression osmotique avec quelque imprécision.

L'autre facteur de la pression osmotique des protéines est le milien ionique des protéines en question, en premier ordre le $p \mathrm{H}$ de cette solution, ou mieux l'écart entre le $p \mathrm{H}$ actuel et celui caractérisant le point isoélectrique [52]. En dehors de ce facteur, ce sont la valence et les qualités des ions de cette solution tampon qui jouent un certain rôle [40].

A quoi se ramènent, au fond, les manipulations pratiquées au cours de la préparation des diverses nourritures infantiles?

L'addition d'eau au lait diminue, dans celui-ci, la concentration des protéines et par conséquent le nombre des particules osmoactives. L'écrémage et la centrifugation agissent de la même manière en entraînant, avec la graisse séparée, toujours quelques quantités de protéines. Les effets de l'acidification sont maxima au point isoélectrique de la caséine $[52,71,102]$. Nous faisons remarquer que les conditions de l'acidité dans les babeurres médicaux, in vitro, et dans le contenu stomacal, après l'ingestion d'un mélange lacté, in vivo, correspondent à la zone isoélectrique de la easéine $(p H 4,7)$. En deçà et au-delà de ce point, la pression osmotique des protéines va augmenter; en pareilles circonstances cependant, la pression est, en présence des ions calciques, toujours plus basse, même dans les zones allant vers l'alcalinase [22,52]. Le pouvoir coagulant, rétractant, déshydratant, acidifiant des ions " $\mathrm{Ca}$ " est bien connu en pathologie générale et en pharmacologie. Toutefois, pour que ces ions deviennent actifs, il faut qu'ils soient ionisés [74]. Dans le lait normal cependant, milieu légèrement acide, c'est la forme non-diffusible, c'est-à-dire non-ionisée de la chaux qui prédomine [70]. Mais il va sans dire que toute acidification du lait est accompagnée d'une ionisation consécutive du calcium, grâce à la solubilisation des phosphates calciques en suspension. C'est ce qui arrive dans l'intérieur de l'estomac où le Ca ionisé va servir ensuite à la phase de coagulation lors de l'emprésurage [74]. C'est ce qui arrive, in vitro, au cours de l'acidification spontanée ou provoquée. On ajoute d'ailleurs des sels ealciques au lait immédiatement dans le procédé de Moll et de Lesné $[49,50]$. L'ébullition du lait en présence de sels calciques exerce habituelle- 
ment une floculation plus ou moins fine et donne lieu à une marche vers l'acidité. Enfin, la présence des ions calciques est assurée d'une manière géniale dans le mode de préparation du babeurre recommandé par A. B. MARFAN qui fait réalcaliser le babeurre par addition d'eau calcique. En ajoutant des combinaisons calciques au lait, on doit compter sur une action osmo-dépressive sinon précipitatrice, effectuée par celles-ci. L'action de la chaleur [87] fait coaguler les albumines dans le lait, ce qui leur enlève toute faculté d'adsorber l'eau, les déshydrate et annihile leur rôle initial joué dans la pression osmotique des protéines. On a également signalé que la solubilité du caséinate calcique diminue, aux températures élevées, ce qui va abaisser leur pression osmotique en faisant diminuer leur dissociation [73]. En ce qui concerne les coupages du lait, on ajoute certaines décoctions au lait dans le but de l'enrichir en colloïdes protecteurs. Cette conception concernant le rôle des colloïdes ajoutés (farines, bouillons de riz, etc.) est à réviser. On peut penser diamétralement le contraire. Ce qui est apparemment beaucoup plus probable, c'est que les matières dextrinées et gommeuses iront s'emparer de l'eau initialement fixée aux protéines du lait et elles vont déshydrater, par cette voie, ces dernières. Quoi qu'il en soit, il est bien connu que les colloïdes sont capables tantôt de stabiliser, tantôt de faire floculer les autres colloïdes présents, car ils gonflent (absorbent l'eau) parfois aux dépens des autres colloïdes (en les déshydratant) et font diminuer la finesse de leur dispersion. C'est ici qu'il convient de faire appel à l'exemple des matières gluténiques de la farine, lesquelles ont des liens de parenté avec la caséine. Le fait que la dextrine et les substances gommeuses peuvent les déshydrater, reste sans aucun doute puisqu'il a été maintes fois affirmé (théorie du pain, cfr. Sohmorl, Moos et Kosmin). D'autre part, on peut facilement observer l'abaissement du nombre des gouttelettes lorsque l'on ajoute au lait des décoctions mucilagineuses ou de la farine de gruau, tandis que le nombre des gouttelettes du lait non préparé surpasse toujours celui de l'eau distillée. Quelques auteurs, comme Schaps et Spolverini [98], ont recommandé l'addition de la gélatine au lait des nourrissons. On sait que l'addition de gélatine à des doses convenables peut provoquer la floculation visible du caséinate calcique [87], équivalant à son osmo-inactivation - puisque les substances en suspension sont dépourvues de toute propriété osmotique. La finesse de la dispersion des protéines peut être abaissée encore en leur ajoutant des cristalloides. PAutr et Rona faisaient remarquer, il y a longtemps, que la présence des sucres facilite le procédé de la gélification de la gélatine [40]. C'est peut-être là que se trouve l'explication du rôle des véhicules sucrés, de certains mélanges de laits enrichis d'hydrates 
de carbone, tels le "Du-bo " de Schick, la prescription de Bessau, le Caro Corn syrup, de Mackim Marriott, ete.

La finesse de la dispersion des protéines est ensuite influencée par de faibles doses d'acide tannique (sous la forme du café, du thé) et par l'addition d'alcool (fréquemment ajouté au lait sous forme de rhum et cognae dans le but de rendre le lait plus tolérable pour quelques malades adultes).

Un des agents les plus actifs qui modifient fortement l'état colloïdal des protéines est la présure. L'emprésurage constitue un mécanisme physiologique destiné à rendre possible la coagulation. et l'osmo-inactivation de la caséine dans un milieu d'une acidité relativement faible. La précipitation de la caséine en milieu acide, au-dessus du point iso-électrique est en quelque sorte possible mais toujours imparfaite. La présence de la présure, par contre, élargit la latitude de l'acidité efficace et elle assure une coagulation forte et efficace entre $p H$ 6,0 et $p$ H 6,5. L'osmo-inactivation de la caséine du lait va être assurée ainsi, même aux $p H$ plus élevés $(7,5)$.

La discussion détaillée de la chimie colloïdale de l'emprésurage, suivant l'excellente ouvre de $\mathrm{Ch}$. Porcher [74], nous fait comprendre la totalité de ce mécanisme, en éclairant la structure du lait au point de vue colloïdal. En résumé, la présure est à considérer comme un ferment protéolytique, produisant le clivage; la paracaséine est toujours insoluble en présence des concentrations des ions $\mathrm{H}$ et Ca. Le produit de l'emprésurage : le caillé, représente un des principaux moyens pour la diététique infantile sous la forme des laits de pégnine, lait caillé de Heim, laits albumineux de Finkelstein-Meyer, ainsi que sous la forme de quelques spécialités desséchées du commerce.

C'est à l'aide de ce mécanisme que l'organisme satisfait à la tâche de l'osmo-inactivation de la caséine. Ce mécanisme fait usage de la présure (ferment-lab), lors d'une certaine acidité et sur l'intervention des ions $\mathrm{Ca}$. La cuisine utilise, en dehors de ces moyens, l'action de la chaleur et encore d'autres manœuvres, avons-nous vu précédemment. C'est ici qu'il convient de mentionner les procédés de la condensation et du desséchement du lait $[29,55]$, lesquelles opérations abaissent considérablement la finesse de la. dispersion des colloîdes. Le degré de la dispersion des protéines du lait reconstitué variera selon la technique utilisée. L'emploi du lait desséché est très répandu en diététique infantile et nous avons, à ce sujet, un nombre considérable de recherches (A. B. MARFAin [55], Roume [85], Gireaux [29], Siranno et Rienzo, Lendrich, Burg et Koppejan [11], Fouassier [23], Génin [28], Howat et Wright [37], Bohn [6], Gautier, Henry et Perroulaz [26], etc.). 
Revenons enfin à la question relative au but de l'osmo-inactivation de la caséine dans les mélanges lactés divers.

Le sol "caséine" - par suite de son osmo-activité. - est un agent purgatif nature]. Une pareille substance, du moinś d'un mode d'action pareil, est le lactose comme suere peu diffusible [67]. Certainement, le lait renferme encore d'autres substances naturelles purgatives.

Or, les manipulations de la technique culinaire ont pour but, du moins en ce qui concerne les protéines, d'anéantir la pression "oncotique ", c'est-à-dire de les faire rétracter, de supprimer leur gonflement, ce qui aura pour effet l'inactivation de la propriété laxative liée à l'état hydrophile.

Il est, en effet, très facilement possible d'obtenir des protéines à l'état osmo-inactif. Toutefois, cette inactivation n'est jamais parfaite et n'est réalisable que dans certaines conditions spéciales et exceptionnelles. Nous rappelons que cette osmo-inactivation comprend un abaissement total de la surface existant entre les particules de caséine et l'eau, et exige une séparation complète du solvant et de la substance dispersée. Ce sont surtout les conditions défavorables à l'emprésurage et à l'acidification "provoquée " qui les rendent imparfaites [74].

En ce qui concerne l'emprésurage, ce sont le " rattrapage ", l'apport d'alcalins, puis le taux élevé des albumines et globulines, enclins à retarder l'emprésurage, qui vont nous intéresser [74] (laits du type colostral). Ces conditions susdites ont pour conséquence non seulement le ralentissement ou la suppression de l'emprésurage, mais la provocation fréquente de diarrhées.

L'acidification provoquée mal conduite, c'est-à-dire l'acidification " brusque " dérange la préparation d'une caséine isoélectrique pure, puisque l'acide n'est pas capable de pénétrer dans les plus intimes parties du coagulum, lesquelles échappent à la neutralisation. L'imperfection de l'acidification s'annonce par une forte teneur en matières minérales [74], etc. C'est peut-être là, dans la divergence de la technique préparatoire, que se trouve l'explication des résultats discordants [63] et inconstants signalés par quelques cliniciens qui ont réexaminé la valeur clinique de quelques prescriptions lactées préconisées par d'autres [63].

Ce sont des discordances dans les modes opératoires pratiqués qui font diverger les résultats obtenus. Par contre, il y a quelques prescriptions qui réalisent, d'une façon très raisonnable, les exigences que nous venons d'indiquer. Les publications classiques de Köppe [44] relatives au babeurre mettent précisément en évidence que cet auteur cherchait à obtenir une caséine isoélectrique passablement déminéralisée. La plupart de ces formules sont 
intéressantes. Jetons un coup d'cil sur la formule de babeurre recommandée, il y a longtemps, par les auteurs hongrois BEREND et HaInISS, qui se prépare actuellement selon la modification du professeur HaIniss.

La méthode du professeur HaINISS [35, 78] comporte plusieurs opérations successives destinées à provoquer des modifications qui suffisent - à mon avis - à réaliser les altérations colloïdales désirées. Ce lait partiellement écrémé, acidifié, enrichi en hydrates de carbone est dénommé " babeurre artificiel " et se prépare dans une cuisine quelconque, très aisément. Les modifications apportées au lait sont : l'abandon du lait cru au repos pendant une heure et demie jusqu'à 4 heures, s'accompagnant d'une acidification spontanée, l'écrémage partiel, l'ébullition après avoir ajouté des saccharo-colloïdes, enfin la perfection de l'acidification, encore incomplète, par addition d'une faible quantité d'acide lactique dilué.

Ayant résumé les effets osmo-dépressifs de quelques manipulations pratiquées à la euisine, revenons encore à la "manipulation " exécutée par l'organisme, c'est-à-dire à l'emprésurage qui se déroule, comme nous l'avons déjà indiqué, dans un milieu légèrement acide [25], en présence d'ions calciques. Le mécanisme spécial de l'emprésurage chez le veau montre déjà la grande importance particulière de cette fonction singulière. Mais, en dehors de tout cela, ce qui reste toujours curieux à constater e'est que l'organisme se contente d'une acidité stomacale légère, laquelle ne réalise chez le nourrisson, presque jamais, les conditions convenables pour une digestion peptique [59] $(p \mathrm{H}=1,7)$ puisque l'acidité du suc gastrique, après l'ingestion d'un aliment lacté, ne dépasse presque jamais la zone isoélectrique de la caséine $(p \mathrm{H} \mathrm{4,7)}$ [25].

Mais ces conditions correspondent, par contre, parfaitement à celles d'une osmo-inactivation par précipitation, c'est-à-dire par coagulation des protéines. Par conséquent, il est aisé de concevoir l'utilité du fait que l'acidité du contenu gastrique du nourrisson corresponde au point isoélectrique de la caséine et tout porte à ne pas considérer ee degré d'acidité comme un simple fait dû au hasard, occasionné par l'intensité du tamponnement à ce point précis, mais on peut - à l'encontre de cette opinion émise par P. GyörGY [34] - y reconnaître à juste titre une sorte d'utilité. On va comprendre également pourquoi nous nous contentons, en ce qui concerne les babeurres et les laits acidifiés, d'une acidification relativement faible : cette acidité facilite, après l'ingestion et après l'intervention du suc gastrique, l'approximation du point isoélectrique.

La coagulation de la caséine semble être d'une plus grande importance que celle de la peptisation, du moins dans l'estomae et en cas de troubles alimentaires. Est-ce à dire qu'il convient de 
n'employer que des protéines qui sont privées de leur solvant "eau "? Bien au contraire. Dans les cas où les troubles de l'échange de l'eau sont absents, un certain degré de gonflement des protéines sera toutefois indispensable, même désirable, pour rendre possible les fonctions peptiques.

En résumé, d'après notre conception, les agents osmo-dépressifs sont: la dilution, en quelque sorte l'écrémage, l'ébullition, l'addition d'acides, de sels calciques, de quelques cristalloïdes (tels le sucrage), de colloïdes (comme le bouillon de riz, etc.). Les facteurs, qui augmentent par contre la pression osmotique des colloïdes dans la préparation des nourritures, sont :

10 L'apport d'un alcalin à cathion mono-valent $(\mathrm{K}, \mathrm{Na})$, qui convertit le caséinate calcique médiocrement gonflé en caséinate de soude fortement gonflé. (La soupe de Keller [54].)

$2^{\circ}$ Le caractère colostral du lait avec un taux élevé en albumines et globulines.

$3^{\circ}$ Finalement, l'omission du délayage (laits concentrés). Les laits étendus auront toujours une pression colloïdo-osmotique plus basse que les laits concentrés - et, à plus forte raison - que les laits enrichis en protéines hydrophiles, parfois surabondamment [70].

Les dernières conditions ont été souvent accusées d'une action purgative.

Or, si l'on a assimilé les colloïdes lyophiles aux solutions salines concentrées au point de vue de la chimie physique, et si l'on a pu pousser cette analogie plus loin, dans les domaines de la pharmacologie des purgatifs, consacrons encore quelques mots à la théorie des diarrhées cholériformes (intoxications alimentaires) établie par Paul Herm, cé mémorable savant hongrois.

Paul HeIm, dans sa théorie suggestive sur la pathogénie de l'intoxication alimentaire, arrive à constater, que les solutions hyper-toniques administrées aux nourrissons sont capables d'ouvrir la barrière hépatique (Lebersperre, de Pick, Mauthner et Molitor), ce qui empêche le rôle antitoxique du foie. Or, si l'on assimile les solutions salines concentrées aux solutions albumineuses "quasi hypertoniques ", on est amené à leur conférer tantôt un rôle toxique, tantôt un rôle siccatif.

Ayant établi, entre les solutions protéiques et les solutions salines concentrées, quelques analogies probables (osmo-anomalies, puissance à attirer l'eau, puissance osmo-laxative, faculté d'ouvrir la barrière hépatique), nous demanderons finalement quelques renseignements sur l'osmo-mécanisme des sécrétions gastriques. Les anciennes recherches du savant italien, Allaria [2], à ce sujet, ont démontré que l'ingestion de solutions hypertoniques adminis- 
trées aux nourrissons, en séries expérimentales, s'accompagne constamment d'un délayage parallèle de ces dernières dans l'estomac, ce qui menait cet auteur à soupçonner l'existence de quelques facteurs osmotiques influençant la production du suc gastrique. En même temps, H. Stöltzner [99] exécutait des mensurations relatives à l'abaissement cryoscopique des nourritures alors usitées, et, tout récemment, HoLtz pratiqua cette méthode pour examiner quelques saccharo-colloïdes utilisés dans l'alimentation artificielle. Ces essais destinés à explorer les origines osmotiques de la production du suc gastrique, en prenant pour base l'abaissement cryoscopique, ont toutefois échoué, à mon avis, faute d'avoir fait intervenir la pression osmotique des protéines.

Nous ne pouvons pas terminer sur ce sujet sans dire quelques mots des travaux de Hoffmann et Rosenbaum [36]. Ces auteurs soutenaient - également sans faire intervenir, dans leur travail, un rôle quelconque de la pression osmotique des protéines - que la sécrétion gastrique excédente, sous l'influence du lait de vache - mais absente après l'administration de lait maternel -, est bien liée au taux élevé des matières protéiques dans le lait de vache. La sécrétion gastrique excédente est due, selon ces auteurs, à la présence de la caséine. Mais cet excédent de la sécrétion gastrique est absent si on nourrit les nourrissons à l'aide de protéines, en milieu acide ou à l'état sec (osmo-inactives, à mon avis).

Les discussions physico-chimiques relatives à la consistance des matières fécales - en plein accord d'ailleurs avec notre conception - dépassent le cadre de ce travail. Nous avons, à ce sujet, un nombre considérable de recherches, par YlPpö, DoRLendourT et Lavandon [18], Hainiss, Stöltzner, Carthagena, Scheer, et Müller [88], Davidsohn, ete.

Les premiers auteurs qui se soient, sciemment, occupés des questions de la dispersion dans la théorie et la pratique de l'alimentation infantile, furent peut-être Rosenstern et LAUTER [80]. Au cours de l'évolution de la chimie physique et de la science des colloïdes se présentèrent bientôt plusieurs auteurs qui ont envisagé les questions diverses de l'alimentation sous ce point de vue (Spolverini [98], Ullmann, Ziegelmeyer, Scheer, Courtney et Brown [16], Demuth [17], Freudenberg [25], Wahlmann, ete.). Parallèlement à la science laitière industrielle, la pédiatrie ne tardait pas à entreprendre également des examens à l'ultramicroscope, entre autres, pour caractériser l'état colloïdal des matières protéiques du lait et diverses autres recherches sur le milieu ionique.

Les chercheurs, qui examinaient le rôle du milieu ionique, négligeaient fréquemment lè rôle déterminant de celui-ci pour les 
changements colloïdaux des protéines. Des auteurs qui, inversement, faisaient jouer un rôle autonome aux modifications colloïdales des protéines, sous-estimaient parfois la haute portée de la correction des rapports ioniques.

Il y a toutefois des auteurs, comme Schiff et Mosse [89], qui, dans leurs excellents travaux sur les laits acidifiés, reconnaissent le rôle important de la corrélation des matières en dispersion moléculaire et en dispersion colloïdale et qui attribuent les effets bénins de ces laits modifiés à tout l'ensemble de ces facteurs. C'est cette corrélation, cet ensemble, qui réalise les conditions convenables de l'état colloïdal nécessité.

En caractérisant les matières dissoutes, on caractérise en même temps le solvant même. Ce n'est pas la connaissance exclusive des premières qui pourra intervenir en vue de la solution définitive de la question, mais il faut sans doute faire aussi une part considérable au solvant.

Les modifications de l'état de l'eau introduite - qui se traduisent par les modifications de la pression "oncotique" des nourritures sont, pour l'échange hydrique, d'une haute importance. Dans le cas donné, comme dans les cas des babeurres et compléments, on peut montrer, que les opérations culinaires détachent l'eau fixée primitivement, des colloüdes, et la rendent ainsi disponible pour les besoins de l'échange hydrique.

\section{LITTÉRATURE}

[1] Achard. L'œdeme brightique, 1929, p. 127.

[2] Allaria. Jahrbuch f. Kinderheilk, vol. LXVI, 259.

[3] Ballovitz. Le Lait, 1935, p. 281.

[4] Behrendt. Zeitschr. f. Kinderhk, 1922, 209.

[5] Belle, Le Lait, 1936, 13.

[6] BонN. Nourrisson, 1936.

[7] Brennemann. Journal Am. Med. Assoc., 1913, p. 575.

[8] - Journal Am. Med. Assoc., 1929.

[9] Briggs. Journal of Phys. Chem., 1932.

[10] Budhanan-Loevmann. Le Lait, 1931, 281.

[11] Burg-Koppejan. Le Lait, 1935, 68.

[12] Caserio. Annal d'Igiene, 1934.

[13] Cherbuliez-Meyer. Helv. Chim. Acta, 1933.

[14] Chi Che Wang Wood. Amer. Journal Dis. Childr., 1930, 787.

[15] Cosmovicr. Comptes rendus Société Biol., 1925.

[16] Countney. Canad. Med. Assoc. Journal, 1928.

[17] Demuth. Ergebn. d. Inn. Med. u. Kinderhk., 1926, 29, 90.

[18] Dorlencourt-Lavandon. Nourrisson, vol. XIX, 1936, p. 147.

[19] Duzar. La funzione del fegato Jemma Vol.

[20] Faber-Hadden. Ref. Zentralb. $f$. Kinderh, 14, vol. CCCLXXXVI.

[21] Fionentini. Riv. Clin. Ped., 1931. 
[22] M. H. Fischer. Die lyophilen Kolloide, Steinkopff, 1935.

[23] Fouassier. Le Lait, 1924, 366.

[24] Friedenthat. Münch. Med. Wschr., 1911, p. 2385.

[25] Freudenbera. Physiologie u. Pathologie der Verdaung im Säuglingsalter, Springer, 1929.

[26] Gautier, Henny, Perroulaz. Rev. Franç. de Péd., 1936.

[27] Gerstley. Amer. Journ. Dis. Ohildr., 1933, p. 538 et 554.

[28] Génin. Le Lait, 1931, 589.

[29] Gireau, Le lait sec en diététique infantile, Paris, Diss, 1932.

[30] Gonce, Templeton. Amer. Journ. Childr., 1930, 265.

[31] Grimmer, Weigmann, Winkler. Handb. der Milchwirtscháft.

[32] Grimmer. Milchwissensch. Forschg., 1928.

[33] Grulee, Heyworth. Journ. Amer. Med. Ass., 10, 1934, 735.

[34] GYöRGY. Arch. für Kinderhk., vol. LXXII, 1.

[35] HaInIss: Monatschr. f. Khhk., 1921. Ibidem : 1923, XXVI.

[36] Hofmmann, Rosenbaum. Jahbuch $f$. Kinderhk, 96-97-100.

[37] Howat, Wright. Le Lait, 1935, 438.

[38] Hоцм, Wевв, Amer. Journ. Dis, Childr., 1930.

[39] Holwerda : Le Lait, 1935, 784.

[40] Höвer. Physikal. Chemie der Zelle u. Gewebe.

[41] Isvesco. La Presse Médicale, 1925, 788.

[42] JAHR. Monatschr. f. Kinderhk., 42, 287.

[43] Kerpel Fronius, Csapo. Zeitschr. f. Kinderheilk,, 1935, 57.

[44] KöpPE. Jahrbuch f. Kinderheilk,, Bd., 63. - Deutsche Med. W., 1904, 917 et $1909,1059$.

[45] Krenn. Chimie et Industrie, 1929, 105.

[46] Krogh, Nakazawa. Biochem. Zeitschr, vol. CLXXXVIII.

[47] Langstein. D. Med. Wschr., no 23, 1927.

[48] LeOpoLd. Zsehr. f. Khk., vol. II.

[49] Lesné, Cofin. Comptes Rend. Soc. Biol., 1927. Lait, 1929, 89.

[50] Lesné, Lataste et Dreyfuss. Zentralbl. f. Kinderhk., vol, 28.

[51] Lichtwitz, Liesegang et Spiro. Medizinische Kollożdlehre.

[52] J. Loeb. Proteins and the theory of colloidal behavior, 1924.

[53] Lombardi, Boll. Soc. Italian. di Biol. Sperim., 1932.

[54] Mathied. Nourrisson, 1934. Rev. Franç. de Puéricult., 1935.

[55] A. B. Marfan. Nourrisson, 1930, 148; 1930, vol. XVIII, 1; Bull. Soc. Ped., 1928 ; Nourrisson, 1936 ; Amer. Journ. Dis. Childr., 1930, 161.

[56] Marriott, MacKim. Journ. Amer. Med. Assoe., 1937, vol. LXXXIX, 862.

[57] C. MeYer. Monatsch. f. Khk., 1923, p. 433.

[58] P. Meyer, Friedheím. Zeitschr. f. klin. Med., 119.

[59] Michaeurs. Praktikum d. Physikal. Chimie, 1922, Springer.

[60] Moнr, Moos. Molkereizeitung, 1932; Milchw. Forschg., 1930-1933.

[61] Molx. Rev. Franç. Péd. et Nourrisson, 1932.

[62] Kometiani, Milchwirsch. Forschg., 1931, 12, Bd.

[63] Morquio. Amer. Journ. Dis. Child., 1931, 421.

[64] Müller. Zeitschr. f. Khk., vol. XXXVIII, p. 705 ; vol. XXXV, 285.

[65] Nassau, Hendelsohn. Klin. Wochenschr., 1923, 1235. 
[66] Оенмe. Klin. Wochenschr., 1923, 1.

[67] Paffrath. Permeabilitaetsstudien Karger, 1931.

[68] Palmer, Dahle. Journ. of Dairy Science, 1922.

[69] Parker, Spackmann. Le Lait, 1931, 283.

[70] Ре́нu. Presse Méd., 1931, 918; Le Lait, 1932, 603.

[71] Pien, Martin. Le Lait, 1932, 513.

[72] Pien, Baisse. Le Lait, 1936, 20, 921.

[73] Pertzoff. Journ. of Gen. Phys., 1927.

[74] Porcher. Le Lait, 1929-1930, 1931; 1932, p. 993 ; Le lait desséché, vol.,

[75] Ribadeau, Mathieu et Guesde. Bull. Soc. Péd., 1031, no 10.

[76] Rice, Frank, Alton, Markley. Journal of Dairy Science, 1924, 405.

[77] Rietschel. Le Lait, 1929, 652.

[78] Rominger. Richtlinien für die Kost des Kindes, Springer, 1935.

[79] Rona, Gaвbe. Biochem. Zeitschr, 1923.

[80] Rosenstern, Lauter. Zeitsch. f. Kinderheilkunde, vol. XXII, 316.

[81] Rosenbaum. Physiologie und Pathologie des Säuglingmagens Karger, 1925.

[82] Rossi. Bol. Soc. Ital. Biol. Sper., 1930.

[83] Rossi. Le Lait, 1930, 278.

[84] Röokemann. Zeitschrift f. Khk., 1926, 261.

[85] Roume. Gynécologie, 1928, 642.

[86] SALGE. Zeitsch. f. Kinderhk., 1911, 1.2.

[87] Samuelsson. Milchwissenschaftl. Forschg., 1932, 233.

[88] Saheer. Op. cit., no 51, Jb. f. Kinderhk., vol. 104, 143.

[89] Schiff, Mosse. Sauere Milchmischungen, Karger, 1924.

[90] Schwarz, Sch. Fischer. Milchwiss. Forschg., 1928, 1935.

[91] Scheuer, Lewis. Journ. of Péd., 1935.

[92] SсhNeck. Le Lait, 1930, 958 ; Milchwiss. Forschg., 1928-1930.

[93] Schlutz, Fetter. Am. Journ. Dis. Childr., 1933-1935.

[94] Schade. Physikalische Chemie in der Inneren Medizin.

[95] Sirks. Le Lait, 1930, 558.

[96] Sмүтн, Hurwitz. Journ. Am. Med. Ass., 1925, 789.

[97] Soxнlet. Münch. Med. Wschr., 1900, 1658.

[98] Spolverini. Le Lait, 1930, 21.

[99] Stöltzner. Jb.f. Kinderhk., vol. LXIII.

[100] Supplee, Beluis. Le Lait, 1924, 358.

[101] Suranyi, Camarella. Il lattante, 1936.

[102] Sutermeister, Brühl. Das Casein, Springer, 1932.

[103] Tapernoux. Le Lait, 1934, 449.

[104] Tapennoux. Le Lait, 1928, pages 686, 795. 916.

[105] Tapernoux. Le Lait, 1936, 832.

[106] Teichert. Le Lait, 1930, 931.

[107] Tria, Zummo. Boll. Soc. Biol. Sper., 1934.

[108] Arch.f. Kinderhk., 109, p. 84.

[109] Vogr. Monatschr. Khk., 30-31.

[110] Henride Weale. Milchwirs. Forschg., 1922.

[111] Whitrier. Journ. Biol. Chem., 1929.

[112] Whittier, Benton. Le Lait, 1928, 825. 